\title{
BREAKDOWN IN FINITE TIME OF SOLUTIONS TO A ONE-DIMENSIONAL WAVE EQUATION
}

\author{
Mokhtar KIRANE and Salim A. MESSAOUDI
}

\begin{abstract}
We consider a special type of a one-dimensional quasilinear wave equation $w_{t t}-\phi\left(\frac{w_{t}}{w_{x}}\right) w_{x x}=0$ in a bounded domain with Dirichlet boundary conditions and show that classical solutions blow up in finite time even for small initial data in some norm.
\end{abstract}

\section{Introduction}

Consider the following hyperbolic system

$$
u_{t}(x, t)=A(u(x, t)) u_{x}(x, t)
$$

where $u: I \times(0, T) \rightarrow \mathbb{R}^{n}$ is a vector-valued function, $A$ is an $(n \times n)$ matrix, and $I$ is an ( bounded or unbounded ) interval. For the Cauchy problem, results concerning global existence and finite life span have been established by many authors. The first who discussed such a problem, in its generality, was John [4] in 1975. He showed that any $C^{2}$ solution of (1.1) blows up in finite time if the initial data $u_{0}(x)=$ $u(x, 0)$ is of compact support and satisfies that $\max _{x}\left\{s^{2}\left|u_{0}^{\prime \prime}(x)\right|\right\}$ is small enough. Here $s$ denotes the length of supp $u_{0}$. His proof makes a crucial use of the local strict hyperbolicity of the system (1.1) in the sense that the eigenvalues of $A(u)$ are real and distinct in a neighborhood of $u=0$. Ta-Tsien et al [14] discussed (1.1) associated with decaying initial data. They proved a global $C^{1}$ solution for the Cauchy problem if, in addition to the local strict hyperbolicity condition, (1.1) is weakly linearly degenerate and the initial data satisfy, for $\mu>0$, that

1991 Mathematics Subject Classification: 35L45.

Servicio de Publicaciones. Universidad Complutense. Madrid, 2000 
$\max _{x}\left\{(1+|x|)^{1+\mu}\left(\left|u_{0}^{\prime}(x)\right|+\left|u_{0}(x)\right|\right)\right\}$ is small enough. They also established a blow up result of $C^{1}$ solutions for non weakly linearly degenerate systems. As they pointed out, their work generalizes their result of [13] to the case of initial data with no compact support but they possess certain decay properties.

For the case $n=2$, the situation is less involved and interesting results have been obtained. For instance, the system of nonlinear elasticity

$$
u_{t}(x, t)=\varphi(v(x, t)) v_{x}(x, t), \quad v_{t}(x, t)=u_{x}(x, t),
$$

has been discussed by Lax [7] and MacCamy and Mizel [8]. In his work, Lax required, in addition to $\varphi>0$, that $\varphi^{\prime}>0$ and showed that classical solutions break down in finite time however smooth and small the initial data are. Whereas MacCamy and Mizel allowed $\varphi^{\prime}$ to change sign and proved a similar result. They also showed, under appropriate conditions on $\varphi$, that there are $x$-intervals, for which the solution must exist for all time even though it blows up for values of $x$ outside these intervals. Messaoudi [11] discussed the following system

$$
u_{t}(x, t)=\alpha(x) \varphi(v(x, t)) v_{x}(x, t) \quad v_{t}(x, t)=u_{x}(x, t),
$$

which models a transverse motion of a string with variable density. $\mathrm{He}$ showed that $C^{1}$-solutions develop singularities in finite time if the initial data are taken with large enough gradients.

For systems with dissipation, we mention the equations

$$
\theta_{t}+c(\theta) q_{x}=0 \quad q_{t}+\sigma(\theta) \theta_{x}=-\lambda(\theta) q,
$$

which describe heat propagation in materials that predict finite propagation speed. This phenomenon is called second sound [1], [2], [9] and [10]. Here $\theta$ is the difference temperature and $q$ is the heat flux. The Cauchy problem was studied by Messaoudi [9] and a blow up result for classical solutions was proved. We should note here that, if $\lambda$ is constant and $c(\theta)=-1$ then (1.4) reduces to a system describing steady shearing flows in nonlinear viscoelastic fluids. This problem was studied by Slemrod [12] and a blow up result for classical solutions has been established. A similar problem was also discussed by Kosinski [6] and Zheng 
[15] and results concerning global existence and nonexistence have been accomplished.

For the higher-dimensional case, it is worth mentioning the result of John [5]. In his work, he considered radial solutions of the threedimensional system of elasticity and showed that classical solutions develop singularities in finite time.

In the present paper we are concerned with a quasilinear hyperbolic system of the form

$$
\left\{\begin{array}{c}
u_{t}(x, t)=\varphi\left(\frac{u(x, t)}{v(x, t)}\right) v_{x}(x, t) \\
v_{t}(x, t)=u_{x}(x, t) .
\end{array}\right.
$$

Note that $\varphi\left(\frac{u(x, t)}{v(x, t)}\right)$ has a dimension of velocity.

We will study (1.5) together with initial and boundary conditions and show that $C^{1}$-solutions blow up even for small and smooth initial data. Our result cannot be directly deduced from the results of [4] and [14] since we do not impose the same conditions regarding the size and the regularity of the initial data (See, for instance, theorem 1.2 of [14] and lemma 3.1 below).

This work is divided into two parts:

In part one we state, without proof, a local existence theorem. In part two, our main result will be stated and proved.

\section{Local Existence}

We consider the following problem

$$
\begin{gathered}
u_{t}(x, t)=\varphi\left(\frac{u(x, t)}{v(x, t)}\right) v_{x}(x, t) \\
v_{t}(x, t)=u_{x}(x, t), \quad \forall x \in I=(0,1), \quad t>0 \\
u(x, 0)=u_{0}(x), \quad v(x, 0)=v_{0}(x), \quad \forall x \in I \\
u(0, t)=u(1, t)=0, v_{x}(0, t)=v_{x}(1, t), \quad t \geq 0
\end{gathered}
$$


MOKHTAR KIRANE AND SALIM A. MESSAOUdI BREAKDOWN IN FINITE TIME OF ...

where $\varphi$ is a $C^{2}$ strictly positive function; i.e

$$
\varphi(\xi) \geq \kappa>0, \quad \forall \xi \in \mathbb{R} .
$$

We also require

$$
u_{0} \in H^{2}(I) \cap H_{0}^{1}(I), v_{0} \in H^{2}(I), v_{0}(x) \neq 0, \forall x \in[0,1] .
$$

Proposition. Assume that $\varphi$ satisfies (2.5) and let $u_{0}$ and $v_{0}$ be given and satisfying (2.6). Then the problem (2.1) - (2.4) has a unique local solution $(u, v)$, on a maximal time interval $[0, T)$, satisfying

$$
u, v \in C\left([0, T), H^{2}(I)\right) \cap C^{1}\left([0, T), H^{1}(I)\right) \cap C^{2}\left([0, T), L^{2}(I)\right) .
$$

Remark 2.1. This proposition is a direct application to the results of Dafermos and Hrusa [3] based on the use of energy estimates.

Remark 2.2. $u, v$ are $C^{1}$ functions by the standard Sobolev embedding theory.

Remark 2.3. The local existence can be obtained even if we have $\varphi(0) \geq \kappa>0$ instead of (2.5). In this case, we only consider initial data satisfying (2.6) with $\left\|\frac{u_{0}}{v_{0}}\right\|_{\infty}$ small enough.

\section{Formation of singularities}

In this section, we state and prove our main result. We first begin with a lemma that gives a uniform bound on $\frac{u}{v}$ in terms of the initial data. Lemma 3.1. Let $\varphi$ be as in the proposition. Then for any $\varepsilon>0$ there exists $\delta>0$ such that for any initial data satisfying (2.6) and

$$
\left\|v_{0}\right\|_{\infty}<\delta, \quad\left\|u_{0}\right\|_{\infty}<\delta
$$

the solution of (2.1) - (2.4) satisfies

$$
\left|\frac{u(x, t)}{v(x, t)}\right|<\varepsilon, \quad \forall x \in[0,1], \quad t \geq 0 .
$$


MOKHTAR KIRANE AND SALIM A. MESSAOUDI BREAKDOWN IN FINITE TIME OF ...

Proof. We define the functions and the differential operators

$$
\begin{gathered}
r:=\ln |v|+\int_{0}^{\frac{u}{v}} \alpha(\xi) d \xi, \quad s:=\ln |v|-\int_{0}^{\frac{u}{v}} \beta(\xi) d \xi \\
\partial_{t}:=\frac{\partial}{\partial t}-\rho\left(\frac{u}{v}\right) \frac{\partial}{\partial x}, \quad D_{t}:=\frac{\partial}{\partial t}+\rho\left(\frac{u}{v}\right) \frac{\partial}{\partial x},
\end{gathered}
$$

where

$$
\rho(\xi)=\sqrt{\varphi(\xi)}, \quad \alpha(\xi)=\frac{1}{\sqrt{\varphi(\xi)}+\xi}, \quad \beta(\xi)=\frac{1}{\sqrt{\varphi(\xi)}-\xi} .
$$

We then compute

$$
\begin{gathered}
\partial_{t} r=\frac{v_{t}}{v}+\alpha \frac{u_{t} v-u v_{t}}{v^{2}}-\rho\left[\frac{v_{x}}{v}+\alpha \frac{u_{x} v-u v_{x}}{v^{2}}\right] \\
=\frac{1}{v}\left[\left(1-\alpha \frac{u}{v}\right) v_{t}-\alpha \rho u_{x}\right]+\frac{1}{v}\left[\alpha u_{t}-\rho\left(1-\alpha \frac{u}{v}\right) v_{x}\right] .
\end{gathered}
$$

We remind that, unless otherwise stated, $\alpha, \beta, \rho$, and $\varphi$ are functions of $\frac{u}{v}$. By noting that $\left(1-\alpha \frac{u}{v}\right)=\alpha \rho$ and $\rho\left(1-\alpha \frac{u}{v}\right)=\alpha \varphi,(3.4)$ gives

$$
\partial_{t} r=0 .
$$

Similar calculations also yield

$$
D_{t} s=0 .
$$

Therefore as long as a smooth solution continues to exist and

$$
\left|\frac{u(x, t)}{v(x, t)}\right|<\sqrt{\varphi\left(\frac{u(x, t)}{v(x, t)}\right)},
$$

$r$ and $s$ remain constant along backward and forward characteristics, respectively; hence

$$
\|r\|_{\infty}=\left\|r_{0}\right\|_{\infty}, \quad\|s\|_{\infty}=\left\|s_{0}\right\|_{\infty} .
$$

To establish (3.6) we note, by virtue of (3.3), that

$$
r-s=\phi\left(\frac{u(x, t)}{v(x, t)}\right),
$$


where $\phi(\tau)=\int_{0}^{\tau} \frac{2 \sqrt{\varphi(\xi)} d \xi}{\varphi(\xi)-\xi^{2}}$ is continuous and strictly monotone, so it admits a continuous inverse $\psi$, at least in a neighborhood of zero. Since $g(\xi)=\varphi(\xi)-\xi^{2}$ is continuous and $g(0)>\kappa$, one can choose $\eta \leq \varepsilon$ such that $g(\xi) \geq \frac{\kappa}{2}$, for all $|\xi|<\eta$ and choose $\gamma>0$ so that $|\psi(\xi)|<\eta$, for all $|\xi|<\gamma$. Therefore as long as $\|r-s\|_{\infty}<\gamma$, we have

$$
\left|\frac{u}{v}\right|=|\psi(r-s)|<\eta \leq \varepsilon .
$$

From the definition of $r$ and $s$, it is easy to see that if $\delta$, in (3.1), is chosen small enough then we get

$$
\left\|r_{0}\right\|_{\infty}+\left\|s_{0}\right\|_{\infty}<\gamma
$$

consequently $\|r-s\|_{\infty} \leq\left\|r_{0}\right\|_{\infty}+\left\|s_{0}\right\|_{\infty}<\gamma$. This implies (3.5); hence

$$
g\left(\frac{u}{v}\right)=\varphi\left(\frac{u}{v}\right)-\left(\frac{u}{v}\right)^{2} \geq \frac{\kappa}{2} .
$$

Therefore (3.6) is established and the proof of the lemma is completed.

Remark 3.1. By using (3.9) and the boundedness of $r$ we conclude, from (3.3) that $\ln |v|$ remains bounded. Therefore, with the above choice of the initial data, $v$ is never equal to zero.

Now, we state our principal result.

Theorem 3.2. Assume that, in addition to (2.5), $\varphi$ satisfies

$$
\varphi^{\prime}(0)>0
$$

and

$$
\frac{v_{0}^{\prime}}{v_{0}}+\alpha\left(\frac{u_{0}}{v_{0}}\right) \frac{u_{0}^{\prime} v_{0}-u_{0} v_{0}^{\prime}}{v_{0}^{2}}>0, \quad \forall x \in[0,1] .
$$

Then there exist initial data $u_{0}, v_{0}$ satisfying (3.1), for which the solution of the problem (2.1) - (2.4) blows up "pointwise" in finite time.

Proof. As usual, we have to derive an ordinary differential inequality with a quadratic nonlinearity for a combination of $u$ and $v$ from which the desired conclusion can be drawn.

For this, we take an $x$-partial derivative of (3.5) to get

$$
\left(\partial_{t} r\right)_{x}=r_{x t}-\rho r_{x x}-r_{x} \frac{\partial}{\partial x} \rho=0
$$


which, in turn, implies

$$
\partial_{t}\left(r_{x}\right)=r_{x} \frac{\partial}{\partial x} \rho=\frac{\varphi^{\prime}}{2 \sqrt{\varphi}} r_{x} \frac{\partial}{\partial x}\left(\frac{u}{v}\right) .
$$

By using

$$
r_{x}=\frac{v_{x}}{v}+\alpha \frac{\partial}{\partial x}\left(\frac{u}{v}\right), \quad s_{x}=\frac{v_{x}}{v}-\beta \frac{\partial}{\partial x}\left(\frac{u}{v}\right)
$$

and substituting in (3.11), we obtain

$$
\partial_{t} r_{x}=\frac{\varphi^{\prime}}{4 \varphi}\left[\varphi-\left(\frac{u}{v}\right)^{2}\right] r_{x}^{2}-\frac{\varphi^{\prime}}{4 \varphi}\left[\varphi-\left(\frac{u}{v}\right)^{2}\right] r_{x} s_{x}
$$

To handle the last term in (3.12), we set

$$
W:=\lambda\left(\frac{u}{v}\right) r_{x}
$$

and substitute in (3.12), to get

$$
\partial_{t} W=\frac{\varphi^{\prime}}{4 \lambda \varphi}\left[\varphi-\left(\frac{u}{v}\right)^{2}\right] W^{2}-\lambda \frac{\varphi^{\prime}}{4 \varphi}\left[\varphi-\left(\frac{u}{v}\right)^{2}\right] r_{x} s_{x}+r_{x} \lambda^{\prime} \partial_{t}\left(\frac{u}{v}\right) .
$$

By using equations (2.1), (2.2), and (3.3) we easily deduce

$$
\begin{gathered}
\partial_{t}\left(\frac{u}{v}\right)=\frac{v\left(u_{t}-\sqrt{\varphi} u_{x}\right)-u\left(v_{t}-\sqrt{\varphi} v_{x}\right)}{v^{2}} \\
=\frac{v\left(\varphi v_{x}-\sqrt{\varphi} u_{x}\right)-u\left(u_{x}-\sqrt{\varphi} v_{x}\right)}{v^{2}}=\frac{\left(\sqrt{\varphi} v_{x}-u_{x}\right)(u+\sqrt{\varphi} v)}{v^{2}}
\end{gathered}
$$

and

$$
s_{x}=\frac{\beta}{v}\left(\sqrt{\varphi} v_{x}-u_{x}\right)=\frac{1}{v} \frac{1}{\sqrt{\varphi}-\left(\frac{u}{v}\right)}\left(\sqrt{\varphi} v_{x}-u_{x}\right) .
$$

Now we choose $\lambda$ so that

$$
-\lambda \frac{\varphi^{\prime}}{4 \varphi}\left[\varphi-\left(\frac{u}{v}\right)^{2}\right] r_{x} s_{x}+r_{x} \lambda^{\prime} \partial_{t}\left(\frac{u}{v}\right)=0 .
$$

By combining (3.14) - (3.16) we arrive, by simple computations, at

$$
\frac{\lambda^{\prime}\left(\frac{u}{v}\right)}{\lambda\left(\frac{u}{v}\right)}=\frac{\varphi^{\prime}\left(\frac{u}{v}\right)}{4 \varphi\left(\frac{u}{v}\right)}
$$


which yields, by a direct integration,

$$
\lambda(\xi)=\varphi^{1 / 4}(\xi)
$$

consequently (3.13) reduces to

$$
\partial_{t} W=\frac{\varphi^{\prime}}{4 \lambda \varphi}\left[\varphi-\left(\frac{u}{v}\right)^{2}\right] W^{2} .
$$

If we choose $\delta$ sufficiently small then the coefficient of the quadratic term in (3.17) remains bounded away from zero by virtue of (3.10), the lemma, and the continuity of $\varphi$ and $\lambda$. So there exists a constant $k>0$ such that

$$
\varphi^{\prime}\left[\varphi-\left(\frac{u}{v}\right)^{2}\right] \frac{1}{4 \lambda \varphi} \geq k,
$$

consequently (3.17) yields

$$
\partial_{t} W \geq k W^{2} .
$$

Therefore (3.18) shows that $W$ ( hence $r_{x}$ ) blows up in a maximal time $t_{m} \leq \frac{1}{k W_{0}}$, if we choose initial data satisfying (3.1) with derivatives satisfying $W_{0}>0$; i.e

$$
\frac{v_{0}^{\prime}}{v_{0}}+\alpha\left(\frac{u_{0}}{v_{0}}\right) \frac{u_{0}^{\prime} v_{0}-u_{0} v_{0}^{\prime}}{v_{0}^{2}}>0, \quad \forall x \in[0,1] .
$$

Remark 3.2. A similar result can be established for $\varphi^{\prime}(0)<0$. In this case we consider the evolution of $s_{x}$ on the forward characteristics; i.e we repeat the same calculations (3.10)-(3.18) with $s$ and $D_{t} s$.

Acknowledgement: The authors would like to express their sincere thanks to the referees for their useful suggestions.

\section{References}

[1] Coleman, B. D., Fabrizio, M. and Owen, D. R., On the thermodynamics of Second Sound in dielectric crystals, Arch. Rational Mech. 80, 135 - 158, (1982).

[2] Coleman, B. D., Hrusa, W. J. and Owen, D. R., Stability of Equilibrium for a nonlinear hyperbolic system describing heat propagation by of Second Sound, Arch. Rational Mech. 94, 267 - 289, (1986). 
MOKHTAR KIRANE AND SALIM A. MESSAOUdI BREAKDOWN IN FINITE TIME OF $\ldots$

[3] Dafermos, C. M. and Hrusa, W. J., Energy methods for quasilinear hyperbolic initial-boundary value problems. Applications to Elastodynamics, Arch. Rational Mech. Anal. 87 (1985), 267--292.

[4] John F., Formation of singularities in one-dimensional nonlinear wave propagation, Com. Pure Appl. Math., 27 (1974), 377 - 405.

[5] John F., Formation of singularities in elastic waves, Lecture notes in Physics 195 (1983), 194 - 210.

[6] Kosinsky, W., Gradient catastrophe of nonconservative hyperbolic systems, J. Math. Anal. 61(1977), 672-688.

[7] Lax, P.D., Development of singularities in solutions of nonlinear hyperbolic partial differential equations, J. Math. Physics 5 (1964), 611-613.

[8] MacCamy, R.C. and Mizel, V. J., Existence and nonexistence in the large solutions of quasilinear wave equations, Arch. Rational Mech. Anal. 25 (1967), 299-320.

[9] Messaoudi, S. A., Formation of singularities in heat propagation guided by second sound, J. Diff. Eqs. 130 (1996), 92-99.

[10] Messaoudi S. A., On the existence and nonexistence of solutions of a nonlinear hyperbolic system describing heat propagation by second sound, Applicable Analysis 73 (1999) 485-496

[11] Messaoudi, S. A., Formation of singularities in solutions of a wave equation, Applied Math. Letters 12 (1999), 23 - 28.

[12] Slemrod, M., Instability of steady shearing flows in nonlinear viscoelastic fluid, Arch. Rational Mech. Anal. 3 (1978), 211-225.

[13] Ta-Tsien Li, Zhou Yi, and Kong De-Xing, Weak linear degeneracy and global classical solutions for general quasilinear hyperbolic systems, Comm. PDE's 19 (1994), 1263 - 1317.

[14] Ta-Tsien Li, Zhou Yi, and Kong De-Xing, Global classical solutions for general quasilinear hyperbolic systems with decay initial data, Nonlinear Anal. T.M.A. 28 \# 8 (1997), 1299 - 1332.

[15] Zheng Yong-shu, Vacuum Problem for the damped p - SY, Studies in Advanced Mathematics, AMS / IP, Vol 3 (1997), 633 - 637.

Mokhtar Kirane

Université de Picardie Jules Verne

Faculté de Math. \& Info

80039, Amiens, Cedex 1, France

E-mail: Mokhtar.Kirane@u-picardie.fr 
Salim A. Messaoudi Math. Sciences Department KFUPM

Dhahran 31261, Saudi Arabia

E-mail: messaoud@kfupm.edu.sa

Recibido: 8 de Febrero de 1999

Revisado: 8 de Marzo de 2000 